\title{
18
}

\section{Automated Calibration using Optimization Techniques with SWMM RUNOFF}

\author{
Shawn Dent, R. Blair Hanna and Leonard Wright
}

The usefulness of a hydrologic model is directly related to its application and how well it is calibrated. Calibration is a subjective exercise where model parameters are adjusted to reduce discrepancies between measured data and modeled predictions. Automated calibration can be used to accelerate the model calibration process, minimize modeler bias, and increase the goodness of fit between measured and modeled hydrographs. During calibration of a complex hydrologic model, it may be difficult to simultaneously adjust predicted output hydrographs to correspondingly match multiple objectives (peak flows, total volume and shape of the hydrograph). Custom programming was used to link SWMM Runoff version $4.4 \mathrm{~h}$ with Palisade's Evolver software to improve model goodness of fit. A small sanitary sewer basin was simulated as part of a collection system rehabilitation pilot program to judge the effectiveness of infiltration and inflow (I/I) removal. A one-month time series of hourly flow measurements were used and calibration was performed with an automated calibration method that applied a genetic algorithm solution technique. Several goodness-of-fit metrics revealed an improved calibration for both pre- and post-rehabilitation flow hydrograph, as well as for projected hydrographs to a design event. This study demonstrates an accurate and cost-effective automated method for model calibration that is not only valuable for repeated model analyses performed throughout a collection system rehabilitation program, but can also be applied to other watershed models.

Dent, S., R. Hanna and L.T. Wright. 2004. "Automated Calibration using Optimization Techniques with SWMM RUNOFF." Journal of Water Management Modeling R220-18. doi:

10.14796/JWMM.R220-18.

(C) CHI 2004 www.chijournal.org ISSN: 2292-6062 (Formerly in Innovative Modeling of

Urban Water Systems. ISBN: 0-9683681-9-0) 


\subsection{Introduction}

Hydrologic models, as commonly used in engineering practice, are mathematical representations of the hydrologic cycle. Hydrologic models come in many forms, and may be applicable to a wide range of spatial and temporal scales. These mathematical representations of the physical world always incorporate some level of simplification through empiricism or the integration of process physics over the spatial and temporal domains. These simplifications introduce error and uncertainty into the analysis, and an adjustment of model parameters is usually performed to reduce error and to better meet the application expectations of the model. This adjustment may be done through manual trialand-error techniques or may be directed by an algorithm.

On the one extreme, the parameter adjustment of a pure empirical model, such as the unit hydrograph, may be accomplished with a wide variety of unconstrained and constrained optimization techniques and therefore trial and error solutions may be avoided (Wright et al. 2001, Wright 2003). On the other extreme, parameters of more complex physically-based conceptual models are traditionally adjusted by manual trial and error solutions. However examples of automated calibration for complex conceptual models are becoming more commonplace as advances in global metaheuristic search routines become more widespread.

Hydrologic models are typically used in storm water applications to estimate the discharge characteristics of a watershed based on the contributing rainfall and the hydrologic attributes of the watershed. The characteristics of a watershed are represented in a model by parameters and relationships that are used to estimate the response of the watershed. Most models have several parameters that can be directly measured or observed (e.g. area of watershed, percent imperviousness) as well as parameters that are not directly observable (i.e. parameters that define ground water flow). Parameters that are not directly observable must be estimated using indirect techniques of matching the model output to historical measured data. The process of adjusting model parameters is called calibration or parameter estimation (Gupta et. al., 1999).

Although the process of simulating a watershed response has been improved through the use of computers, the calibration of hydrologic models is still commonly performed manually using a trial-and-error approach. How well a model is calibrated depends on the sophistication of the model, the modeler's expertise, the model application, and the accuracy and extent of the data (rainfall and flow) that is available. Simple models tend to condense the hydrologic process of interest into a few parameters (e.g. the Rational Method). 
More sophisticated or complex models describe the hydrologic processes with many parameters to better approximate the observed response of a watershed. A complex model is not always a better choice compared to a simple model; this judgment depends on external factors such as the application, the budget of the analysis and the experience of the modeler. An experienced modeler is likely to calibrate a model more accurately and in less time than an inexperienced person because of expert knowledge gained from previous calibration exercises.

The accuracy and extent of data, while many times taken for granted, may also dramatically affect the quality of the calibration. For example most properly installed and operating open-channel flow meters and gauging stations are only accurate to within a five-percent range of measured flows (Stonehouse et al., 2001). Also, if the measured data is only available for a limited period of time, the model will be similarly limited as it will be based on a small window of the hydrologic response of the system. If more extensive data are available and exhibit a wide range of conditions including low and high flow events as well as short and long duration events, a more accurate and precise calibration can be accomplished that may better meet a wide variety of goals. Discussions on the importance of calibration for surface water models can be found in Wright et al. (2001), Wright (2003), Dent et al. (2000), Nix (1994) as well as for ground water models in Hill (1998).

Basic hydrologic models behave simply; parameter adjustments tend to produce predictable model output. Complex models tend to have a large number of parameters to describe hydrologic processes and are based on complex mathematical relationships, which can cause parameters to interact in an unpredictable fashion. To assist the modeler in calibrating complex models, research into the use of automatic calibration techniques began in the 1960s. Since then, advancement has been made in this field due to increased computer speed and more robust and efficient solution techniques. Recent applications of automatic calibration to hydrologic models include Madsen and Jacobsen (2001), Wangwongwiroj et al. (2001), Madsen (2000), Gupta et al. (1998, 1999), Khu (1998), Sorooshian et al. (1998), and Duan et al. (1992).

There are many advantages in applying global optimization techniques to complex hydrologic models. Global optimization techniques, as opposed to manual calibration, may provide the modeler with a closer fit between model outputs and field measurements and may also decrease uncertainty in the process (Doherty and Johnston, 2003). The objectives in applying automatic calibration in this study include:

- time savings - calibrating many basins with a complex model is time intensive and can be cost prohibitive; 
- decreasing bias - automatic calibration can decrease the bias inherent in manual calibration by objectively estimating model parameters; and

- increasing goodness-of-fit - comprehensive parameter estimation will usually increase accuracy and precision of a model.

In this chapter, automatic calibration of the Runoff block of the U.S. Environmental Protection Agencies Storm Water Management Model(SWMM) version $4.4 \mathrm{~h}$ is applied to the hydrologic response of Infiltration and Inflow (I/ I) in a sanitary sewer collection system. Although automatic calibration of SWMM, as applied to general hydrologic scenarios, has been documented by James et. al. (2001), Wan and James (2001), Liong et al. (1991, 1994, and 1995), and Nguyen etal. (2000), little if any applications of automatic calibration have been applied to modeling I/I. This chapter will illustrate the effectiveness of applying automatic calibration to modeling $\mathrm{I} / \mathrm{I}$ by demonstrating the use of a Genetic Algorithm (GA) parameter optimization routine coupled with SWMM, and applied to a sanitary sewer basin in Vallejo, California.

\subsection{Mathematical Models}

Several hydrologic models have been coupled with parameter estimation software to automatically calibrate flows to a specific watershed response. Doherty and Johnston (2003) have recently applied the parameter estimation model PEST (Gauss-Marquardt-Levenberg method) to the Hydrologic Simulation Program Fortran (HSPF). Duan et al (1992), Sorooshian et al, (1993), and Gupta et al $(1998,1999)$ coupled a shuffled complex evolution (SCE-UA) global optimization algorithm to the Sacramento soil moisture accounting(SAC-SMA) model of the National Weather Service River Forecast System (NWSRFS). Madsen and Jacobsen (2001), Madsen (2000), Wangwongwiroj et al. (2001), and Khu (1998) also applied the shuffled complex evolution (SCE) method to calibrate the MIKE SHE, MIKE 11/NAM, MOUSE, and NAM models by Danish Hydraulic Institute (DHI), respectively.

SWMM has also been coupled with several parameter estimation routines documented by Liong et al. (1991, 1994, and 1995). Nguyen et al. (2000) applied the SCE method to the Runoff block of SWMM. Wan and James (2001) and James et al. (2001) have integrated a GA routine in PCSWMM (Computational Hydraulic International) to assist in calibration. The GA routine can be used on any parameters in Runoff, Transport, or Extran. The above studies have applied automatic calibration to either conceptual rainfall-runoffmodels or 
urban runoff models, but this is the first study, to the authors knowledge, that applies automatic calibration techniques to modeling $\mathrm{I} / \mathrm{I}$ in a sanitary sewer system.

\subsubsection{Hydrologic Model}

Modeling I/I is typically not considered a hydrologic process because many commercial sanitary sewer models lump the hydrologic process with the hydraulics and market the package as a hydraulic model. However, wet weather flow estimation is the same for $\mathrm{I} / \mathrm{I}$ in a sewer basin as it is for a watershed. Therefore, version 4.4h of SWMM (Huber and Dickinson, 1988) was used in this study along with automatic parameter estimation techniques to calibrate to measured flows in a sanitary sewer basin.

Specifically, the SWMM Runoff block was used as the hydrologic model for this study. SWMM Runoff is a lumped parameter deterministic model that can be used for continuous simulation (many storms) and is typically referred to as an urban runoff model. However, Runoff also contains a near surface groundwater routine that when coupled with the surface water routine, can accurately simulate the hydrologic response of an urban or rural watershed, or in this case, $\mathrm{I} / \mathrm{I}$ in a collection system. SWMM is described in many sources and will not be discussed in detail in this chapter. A detailed description of SWMM can be found in the literature (Huber and Dickinson, 1988; Nix, 1994; James and James, 2002).

SWMM Runoff estimates the amount of wet weather flow discharged from a basin by translating rainfall into runoff using a non-linear reservoir routine. A physically based non-linear reservoir model provides several advantages over other methods (i.e. non-physically based methods such as unit hydrograph and regression models) when applied to approximate the reduction of wet weather flow in a sanitary sewer basin due to pipe rehabilitation to reduce $\mathrm{I} / \mathrm{I}$. The main advantage is that a physically based model relies on an area parameter to approximate the amount of flow. In modeling I/I the typical area parameter essentially becomes an effective area. The effective area reduction (based on the amount of $\mathrm{I} / \mathrm{I}$ reduction) along with changes in ground water parameters have a variable effect on the hydrograph, as opposed to a constant reduction factor that would be applied in non-physically based methods to approximate I/I reduction (Wright et al., 2001).

Area is only one of the many parameters that can be manipulated in SWMM Runoff to calibrate a model. However, an output hydrograph is typically more sensitive to certain select parameters. Most hydrologic models that are similar 
in complexity to SWMM Runoff typically have several of these calibration parameters. Table 18.1 summarizes the ten calibration parameters that were chosen for this study based on the literature (Nix, 1994; James and James, 2002), and the authors' experiences.

Area is not a calibration parameter in a typical watershed (area is measured directly). However, since the area, or effective area of $I / I$ is not known due to the distributed nature of defects in collection system, this parameter needs to be estimated. To bound this estimate, the effective area must obviously be less than the total area of the sewer basin. For most sanitary sewer basins the effective area usually ranges between $1 \%$ and $10 \%$ of the total area depending on the severity of the I/I. It is because of the distributed nature of $I / I$ that it is essential that a basin be monitored to accurately project I/I.

Table 18.1 SWMM Runoff calibration parameters.

\begin{tabular}{cl}
\hline Parameter & Definition \\
\hline Surface Water & \\
WAREA & $\begin{array}{l}\text { Area (acres) - Effective Area in this case } \\
\text { IMPERV }\end{array}$ \\
WIDTH & Width of overland flow \\
Groundwater & \\
STG & Elevation of initial water table stage \\
BC & Threshold stage for groundwater flow \\
TW & Channel water influence factor \\
A1 & Groundwater flow coefficient \\
B1 & Groundwater flow exponent \\
A2 & Coefficient for channel water influence \\
B2 & Exponent for channel water influence \\
\hline
\end{tabular}

\subsubsection{Optimization Algorithm}

Model calibration was performed using the Evolver software package (Palisade Corp, 2001). Evolver is an Excel add-in that utilizes a GA optimization routine. GAs are a broad class of global search routines called metaheuristics (Glover and Laguna, 1997). Global search techniques are useful for finding high-quality solutions to non-convex and mixed integer problems. Metaheuristics are wellsuited to complex hydrologic model parameter estimation because the underlying functions may be based on discrete or discontinuous, non-convex functions.

This software was chosen for two reasons. The optimization program works seamlessly with Microsoft Excel, which was later linked to Runoff. And, using off-the-shelf software releases the modeler from programming a custom 
optimizer as well as providing the added benefit of upgrading when new versions become available. By capitalizing on a spreadsheet model structure, which provides a link between the hydrologic model and the optimization engine, both programs are leveraged. When either the model or the optimization engine is updated, relatively simple adjustments can be made to accommodate these changes. This structure also allows for the potential "plug-in" of other optimization or risk analysis programs. Further discussion of linking the SWMM Runoff model to an Excel spreadsheet is included later in this chapter.

\subsection{Study Area}

Estimation of wet weather flows in a sanitary sewer basin remains one of the more challenging problems in urban hydrology (Wright et al, 2001). The Vallejo Sanitation and Flood Control District (VSFCD) is currently facing the challenges of constructing and calibrating models that accurately describe $\mathrm{I} / \mathrm{I}$ within their sanitary sewer system. I/I due to wet weather flows, also known as rainfall dependent infiltration and inflow (RDII), can usurp conveyance capacity in a collection system and cause sanitary sewer overflows (SSOs). Thus, an accurate characterization of $\mathrm{I} / \mathrm{I}$ within a collection system model is paramount to developing a capital improvement program (CIP) that will cost-effectively remediate excessive I/I (including SSOs).

\subsubsection{Pilot Program}

VSFCD operates sanitary and storm sewer systems in the City of Vallejo, California. This study concentrates on modeling the wet weather response of sanitary sewer basins in VSFCD's system. The sanitary sewer system experiences excessive I/I during the wet weather season in the San Francisco bay area (typically November through April). This system also suffers from a high ground water table that can be less than two feet below ground level and contributes a constant source of infiltration to the submerged sewers.

A pilot program was conducted by VSFCD as part of an overall CIP. The objective of the pilot program included the identification of test and control basins, pre-rehabilitation flow monitoring, rehabilitation of select basins, and post-rehabilitation flow monitoring to identify the amount of $I / I$ that could be cost-effectively removed. Figure 18.1 illustrates the layout of VSFCD's system (including 160 basins) and the twelve test and control basins within the sanitary sewer system that were calibrated for the pilot program (Carollo, 2003). 
This chapter concentrates on the use of a GA to calibrate pre-rehabilitation and post-rehabilitation wet weather flows discharged from a single test basin. Test Basin III was chosen for this study because it had high-quality pre and post-rehabilitation measured flow data, and a significant portion of the subbasin was rehabilitated (Dent et al., 2003 and Carollo, 2003).

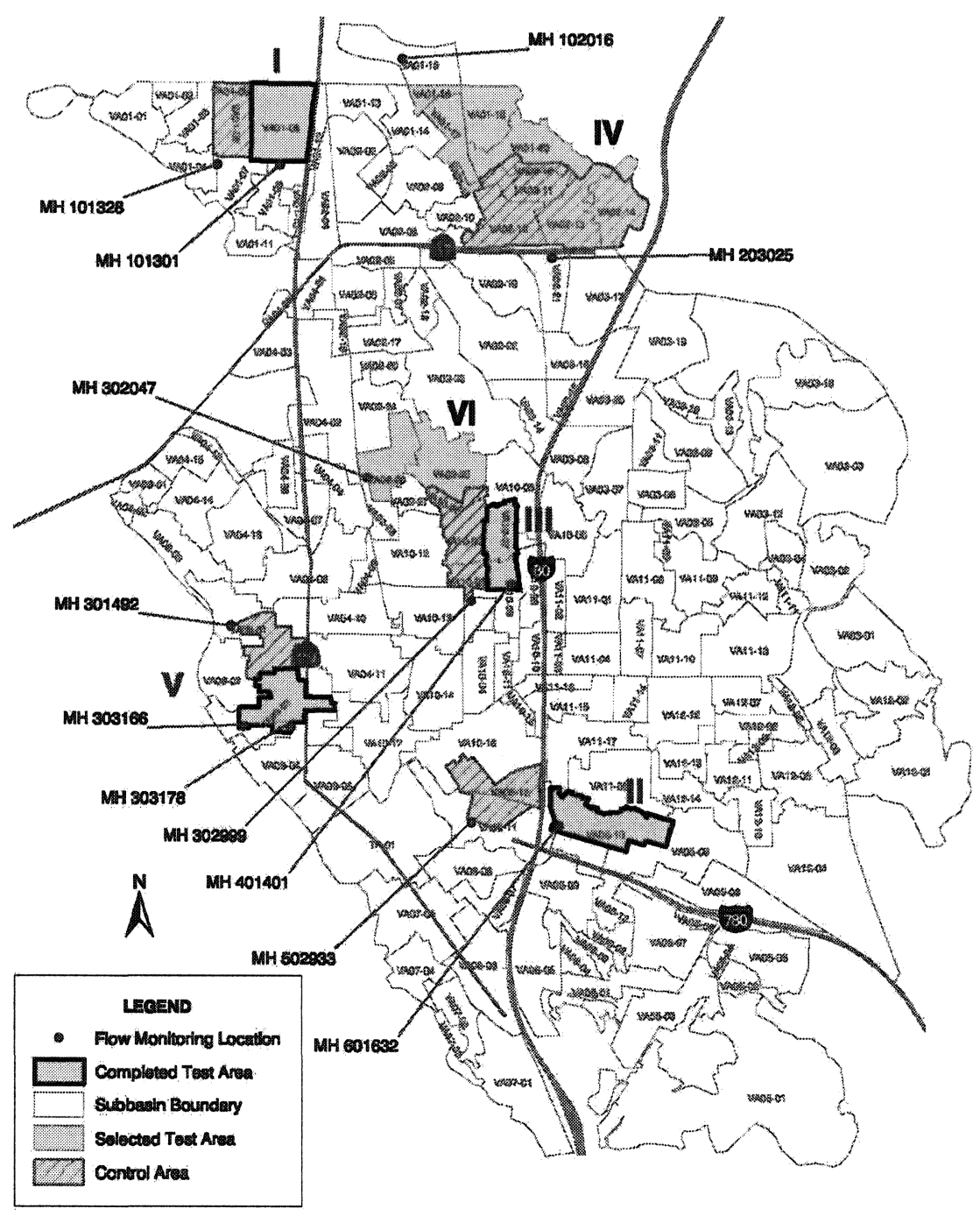

Figure 18.1 Location map of VSFCD including Test and Control basins (see Test Basin III in middle of system). 


\subsubsection{Definition of Infiltration and Inflow (I/I)}

$\mathrm{I} / \mathrm{I}$ as well as RDII has been comprehensively defined in many references and will not be discussed in detail in this chapter. A general definition of $\mathrm{I} / \mathrm{I}$ can be found in ASCE/WPCF (1983). Another definition of I/I, specifically referred to as RDII, can be found in Wright et. al. (2001). This chapter will use the general term of $I / I$ to refer to both the classical definitions of $I / I$ and the more specific definitions of RDII.

I/I are extraneous wet weather flows that enter a sewer system through a variety of sources. Infiltration generally enters a sanitary sewer system through cracks in pipes, joints, or manholes and consists of a delayed, drawn out flow response because rainfall has to seep through the soil matrix before entering the collection system. Inflow is typically contributed by directly connected impervious areas (DCIA), such as catch basins or roof leaders, and contributes an immediate response of flow from incident rainfall. Another source of $I / I$ that acts in a hybrid manor includes sump pumps, which initially collect rain water that infiltrates through the soil matrix, but then discharges an immediate spike of flow to the sewer system. In general, infiltration occurs from subsurface sources, while inflow is mainly contributed by surface sources. An I/I hydrograph response tends to be similar to the response of a watershed that has both urban and rural landuse (e.g. the quick response followed by the drawn out response).

\subsection{Approach}

Generally, the calibration process is the same for any hydrologic model. Figure 18.2 illustrates the automatic calibration process specifically for this analysis. Automated calibration utilizes a search routine (or optimization algorithm) to continuously adjust model parameters until a "good fit" is achieved between the measured and modeled data. The goodness-of-fit is calculated using one or several objective functions.

\subsubsection{Objective Functions}

There are a variety of objective functions that may be used to measure the goodness-of-fit between a measured and a modeled hydrograph. Gupta et. al. (1999) used four objective functions, or error statistics, in testing the calibration of a complex hydrologic model that used a daily timestep. These error estimates 


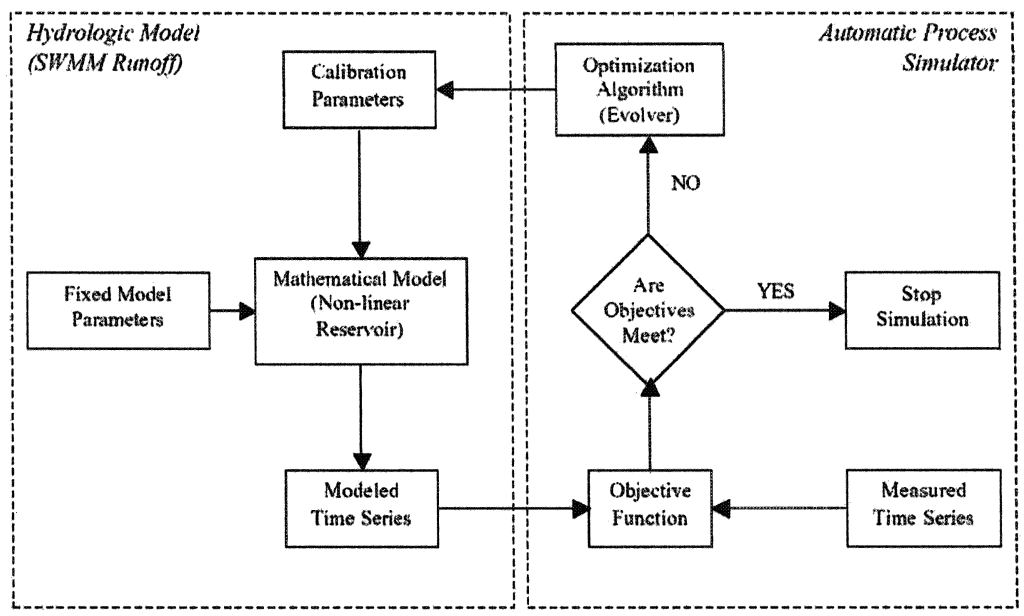

Figure 18.2 Generalized automatic calibration process.

included root mean square error - RMSE (termed DRMS - daily root mean square), bias (termed PBIAS - percent bias), Nash-Sutcliffe efficiency (termed NSE), and persistence model efficiency (termed PME). Madsen et. al. (2001) used RMSE and bias as applied to groundwater level and runoff in the MIKESHE model.

This analysis initially utilized RMSE as the objective function that was minimized using the GA algorithm. This procedure worked well, but it was difficult to explicitly compare one calibration against another since RMSE will vary depending on the length of the time series. Therefore, it is also necessary to examine other objective functions that normalize the errors between simulated and measured time series so that the calibration of any length of time series can be directly compared to another.

Nash-Sutcliffe (1970) first introduced the unmodified coefficient of efficiency. This objective function has been used throughout the years in many hydrologic studies. The unmodified coefficient of efficiency is defined as:

where:

$$
E_{N S}=1-\frac{\sum\left(Q_{S}-Q_{M}\right)^{2}}{\sum\left(Q_{M}-\bar{Q}_{M}\right)^{2}}
$$

$$
\begin{aligned}
\mathrm{Q}_{\mathrm{S}} & =\text { simulated flow, } \\
\mathrm{Q}_{\mathrm{M}} & =\text { measured flow, and } \\
\bar{Q}_{M} & =\text { mean of the measured flow time series. }
\end{aligned}
$$


$\mathrm{E}_{\mathrm{NS}}$ can range from minus infinity to 1.0. Values approaching 1.0 indicate the highest confidence in agreement between simulated and modeled flow time series.

Legates and McCabe (1999) provided a study of a number of indices to test the goodness-of-fit between measured and modeled time series including the unmodified coefficient of efficiency (Nash-Sutcliffe) and the modified coefficient of efficiency along with several other coefficients. Doherty and Johnston (2003) suggested that the modified coefficient of efficiency provides as well if not better measure of the goodness-of-fit between measured and modeled flows than $\mathrm{E}_{\mathrm{NS}}$. The modified coefficient of efficiency is defined as:

$$
E_{M}=1-\frac{\sum\left|Q_{S}-Q_{M}\right|}{\sum\left|Q_{M}-\bar{Q}_{M}\right|}
$$

where:

$$
\begin{aligned}
\mathrm{Q}_{\mathrm{S}} & =\text { simulated flow, } \\
\mathrm{Q}_{\mathrm{M}} & =\text { measured flow, and } \\
\bar{Q}_{M} & =\text { mean of the measured flow time series. }
\end{aligned}
$$

Values that exceed zero illustrate that the model can be considered a better predictor than the mean of the observed data. This analysis initially used RMSE but also reported $\mathrm{E}_{\mathrm{NS}}, \mathrm{E}_{\mathrm{M}}$, and the typical $\mathrm{R}^{2}$ error estimates. Although these error estimates were used for this study, further research should be performed to evaluate which objective function works best for hourly flow data for sanitary sewer wet weather flows.

\subsubsection{Available Data}

Wet weather flows measured in a sanitary sewer system have to be prepared before modeling can take place. Sanitary sewer flows contain both dry weather flow (DWF) as well as wet weather flow (WWF) as described earlier (see Wright et al., 2001). Therefore, a dry weather flow estimate must be first subtracted from the total flow hydrograph to estimate the wet weather flow. This preparation was accomplished by choosing a representative week of DWF and subtracting that repeated week from a month worth of total flow. This process is illustrated in Figure 18.3.

This procedure does introduce error into the resultant WWF hydrograph. The error that results is minimized if the chosen representative week of DWF corresponds well with the DWF that actually occurred during the wet weather 


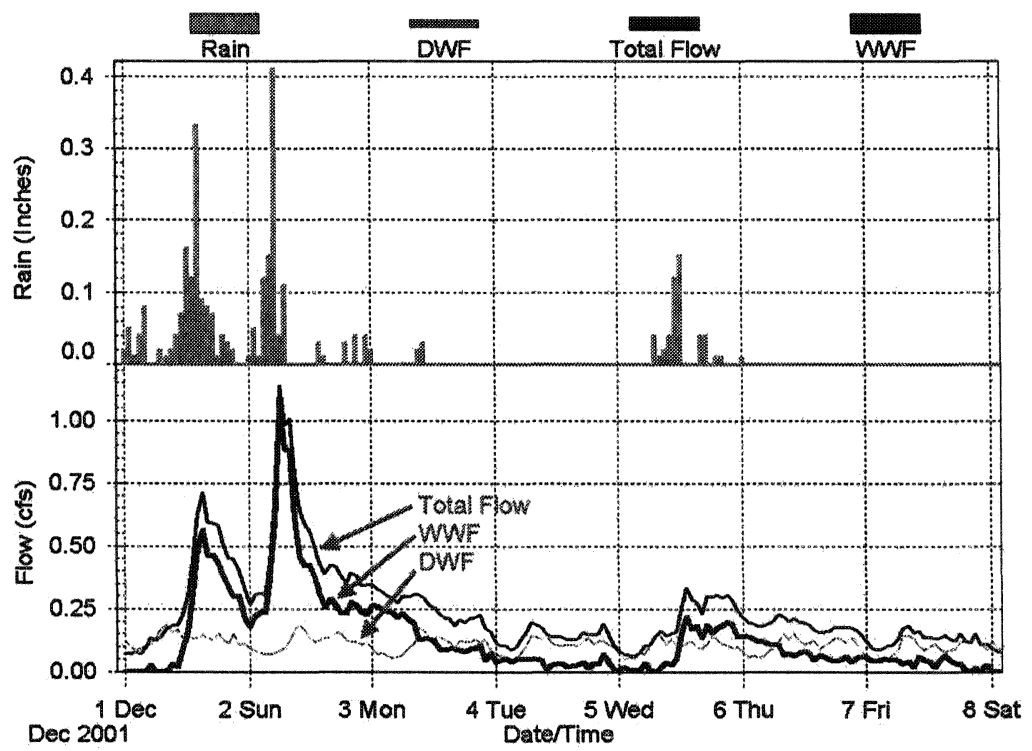

Figure 18.3 Wet weather flow preparation of a week's worth of hourly measured sanitary sewer flow (where DWF is dry weather flow and WWF is wet weather flow).

flow period (however, this can never be truly known). This error can be assessed by examining the WWF hydrograph and identifying if the flows remain positive over the subtracted period and do not exhibit a diurnal pattern along the receding limb of any hydrograph.

Figure 18.3 only shows a one-week period of measured data for clarity. This process was repeated for a months worth of hourly flow for the test basin for both the pre and post-rehabilitation periods. Once the WWFs were separated from the total measured hydrograph, the Runoff block of SWMM could be used to construct a model of the basin.

\subsubsection{Model Construction}

The SWMM Runoff model (version $4.4 \mathrm{~h}$ ) consists of an executable program (DOS-based written in Fortran), text files for input and output data, and a binary output file. This model structure is cumbersome to link with an Excel-based GA. The first attempt at linking the SWMM Runoff model with an Excel spreadsheet (which allows the use of the Evolver optimization program) utilized 
the reading and writing to and from the text input and output files. This process was cumbersome as well as time intensive because of the format of the SWMM text files (paginated with headers).

Ultimately, the SWMM Runoff file was recompiled into a dynamic link library (DLL) which allows relatively direct access of the model by the spreadsheet. The SWMM DLL was modified to produce a custom output file. This file was small and had simple formatting, i.e. it did not contain page headers or error information that might change the file structure from run to run. This file was created during each GA iteration and compared to the measured flow file. The goodness of fit statistics were then returned to Excel and entered into the objective function of the GA. The quality of the solution was quantified by the objective function.

On a Pentium III $833 \mathrm{MHz}$ computer, a single run (one basin run for one month with hourly flows in SWMM Runoff as well as calculation of the objective function in the spreadsheet) takes less than one second. This allows tens of thousands of runs to be completed in a reasonable time frame for a single basin.

Each model run consists of the optimization program choosing values for each of the up to ten calibration parameters. The selection of the new parameter values are based on a random component as well as information from previous high quality solutions. These new variables are then written to the SWMM Runoff input file and the DLL is called from within Excel and run. The simulated time series of hourly flows is then read from the custom text file and compared to the measured flow text file. The objective function is then calculated and returned to the spreadsheet. The Evolver program, running within Excel, tracks each run, the value of each calibration parameter, and the objective function result. Evolver then chooses new parameter values and keeps running until the objective function meets a certain criteria, a set number of model runs are met, or the user halts the process.

It was found during system development that certain parameters could be constrained to improve calibration efficiently. Constraints were included in the model in two ways. Each of the ten parameters was constrained to a range of potential outcomes. This range is defined in Evolver and provides a discrete range (or search space) for the GA to select parameter values. Additional constraints were included in the model due to functional forms of the groundwater equations in SWMM Runoff. James and James (2002) provide an explanation of the groundwater equations used in SWMM Runoff and offer suggestions on how to constrain these parameters to provide a realistic estimation of the groundwater hydrograph. 
The values for the range of each parameter as well as the constraints on the interaction of the parameters are summarized in Table 18.2. The upper and lower ranges summarized in Table 18.2 are based on the authors' experience. The constraints in Table 18.2 are based on the discussion in James and James (2002). The A3 parameter in the SWMM Runoff data file was fixed at zero.

Table 18.2 Parameter ranges and constraints.

\begin{tabular}{cccc}
\hline Parameter & Lower Range & Upper Range & Constraints \\
\hline Surface Water & \multicolumn{3}{c}{} \\
WAREA & 5 & 500 & -- \\
IMPERV & 5 & 95 & -- \\
WIDTH & 10 & 1000 & -- \\
Groundwater & & & \\
STG & 0.1 & 10 & \\
BC & 0.1 & 9.5 & BC $>$ STG \\
TW & 0.1 & 9.0 & TW $<$ BC \\
A1 & 0.001 & 1.000 & A1 $>$ A2 \\
B1 & 0.1 & 9.9 & B1 $>$ B2 \\
A2 & 0 & 1.000 & -- \\
B2 & 0 & 9.9 & -- \\
\hline
\end{tabular}

\subsection{Results}

The results of this analysis are presented based on the calibration to measured flow data as well as projections that are made for a significant wet weather event that occurred outside the calibration time frame.

\subsubsection{Calibration}

Measurement and modeling introduce errors; there is no objective way a unique model solution can be obtained (i.e. there is no "best" solution - also referred to as a non-dominated solution). Calibration is very subjective and several authors have noted that a model can only be calibrated to the amount of data that is available. Even in the case where a significant amount of data exists, modeled estimates will not produce one set of calibration parameters that are best for all situations. This situation is especially true when high and low flow estimates are compared (see Gupta et al., 1998; Madsen and Jacobsen, 2001; Doherty and Johnston, 2003; as well as others). Therefore the terms verification and 
validation are not used in this chapter because it is the authors belief that a hydrologic model is never truly validated, that is, there is no one set of parameters that will apply to all the measured flow data (either past or future measurements).

In calibrating a hydrologic model, it is also important to take into consideration rainfall variability. Rainfall variability introduces error into the modeling process because rain is measured at select points within a watershed. Rain gages (i.e. tipping buckets) are point estimates of rainfall. Even radar rainfall estimates are still estimates although radar can dramatically increase the accuracy of measured rainfall across a watershed. Although rainfall variability is hardly discussed in the literature reviewed, it is a measurement error and can significantly affect the calibration of a hydrologic model.

Any model can be further calibrated if additional measured data are collected, but time and costs constrain modeling efforts in typical sewer system projects. For the purpose of this study, a month of measured hourly flow and rainfall data were used to calibrate the pre and post-rehabilitation models. Both the pre and post-rehabilitation periods contained significant high and low flow events. It was especially important for this analysis that high flows be represented because the reduction in I/I peak flows reduces the amount of downstream conveyance improvements that may be necessary. However, representing the entire hydrograph accurately is also important, from a volumetric standpoint, especially if storage facilities are considered in place of reducing $\mathrm{I} / \mathrm{I}$.

The Evolver calibration procedure was applied to the SWMM Runoff model using ten calibration parameters. The calibration was completed for two sets of data; one set for the pre-rehabilitation flow data, and one set for the postrehabilitation flow data. Each model was allowed to run for approximately 35,000 simulations (which took about $8 \mathrm{~h}$ of computational time). Most times the RMSE (the objective function used to judge the calibration) would be minimized well before the 35,000 simulations were complete. However, running the model over this period virtually guaranteed the minimum value would be reached. Figures 18.4 and 18.5 illustrate the results of the pre and post-rehabilitation calibrations.

\subsubsection{Projections}

Applying pre and post-rehabilitation flow data to this study allowed for a unique comparison when applying automatic calibration. For example, when the same rainfall event(s) are applied to the calibrated models, the difference in the two 
hydrographs should only be a result of the reduction in flow due to rehabilitation of the sanitary sewer system (e.g. removing I/I sources). Therefore, a major rainfall event, which included significant antecedent conditions from previous storms, was used to compare the resultant hydrographs for each model.

There are several different types of design events that can be selected to analyze the capacity of collection system (Dent et al., 2002). An event was selected, from a longterm record, during a wet season that had many storms and resulted in much higher than average total rainfall for the wet season (November through April in Northern California). Rainfall from an entire wet season were run through each model (pre and post-rehabilitation). The results for the selected event that occurred in March 1983 were then compared. The March 1983 event was previously determined to be a 5-y design event (Carollo, 2003).

Results from these two models presented an interesting dilemma. Initially the two hydrographs (pre and post) were inconsistent. For example, the peak flows were less for the post hydrograph, but at some points along the time series, the flows along the recession limb of the post-rehabilitation hydrograph would be greater than the flows for the pre-rehabilitation hydrograph. These

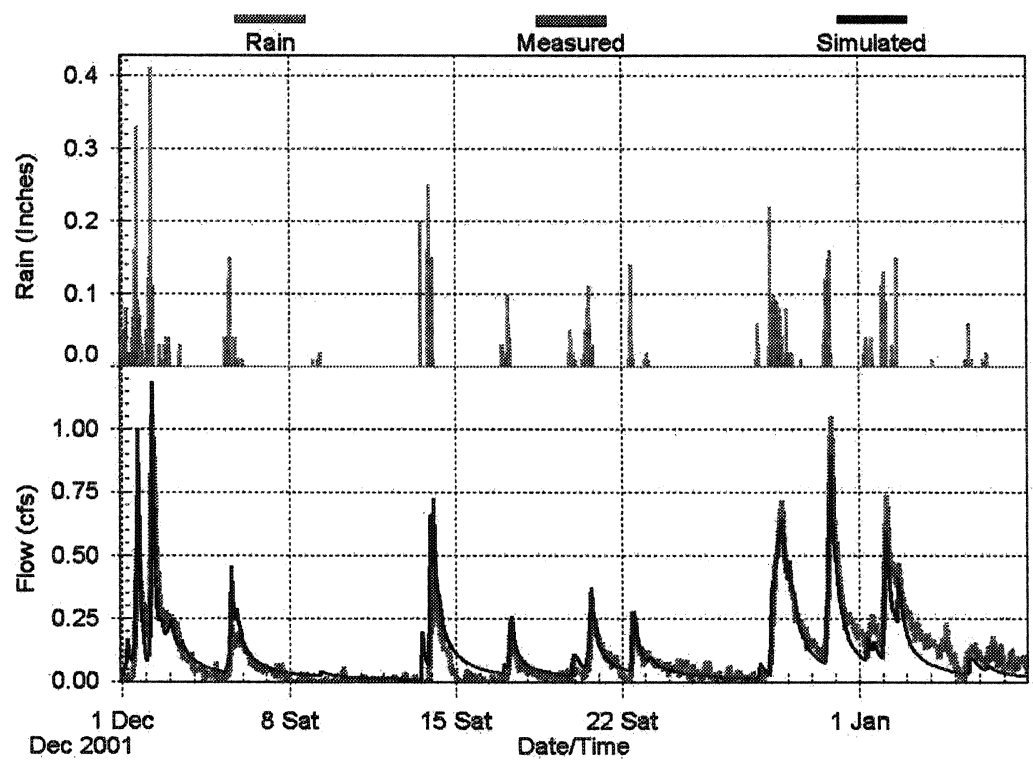

Figure 18.4 Pre-rehabilitation calibration hydrographs (hourly rain and flow). 


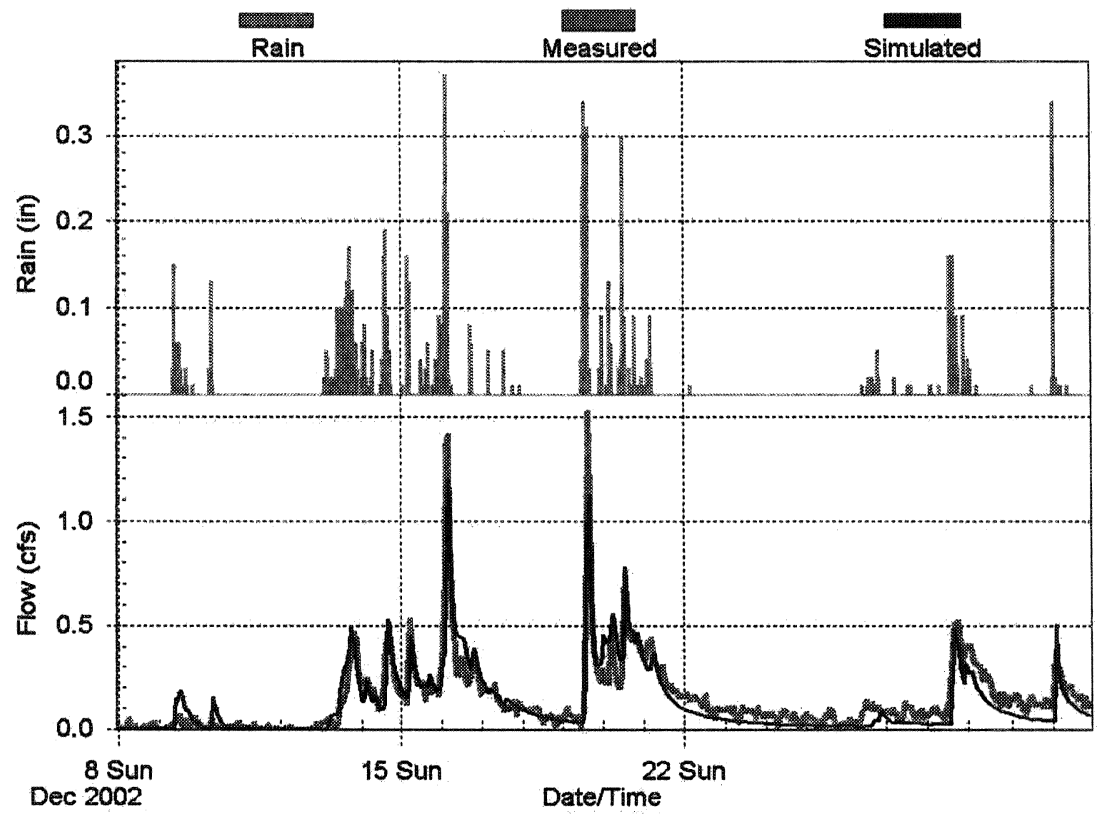

Figure 18.5 Post-rehabilitation calibration hydrographs (hourly rain and flow).

results illustrated that there was an inconsistency in the parameters that were estimated for each of the models (pre and post-rehabilitation). This inconsistency is an example of why model results are not truly validated since the calibrations were optimal from an overall error standpoint, but when used to project results for a single design event, were not optimal to provide results that were consistent.

To correct this inconsistency, the percent impervious (IMPERV) and width (WIDTH) parameters were fixed so they were equal in both models while the other parameters were allowed to vary according to the ranges and constraints summarized in Table 18.2. After testing many combinations, a value of $35 \%$ was set for the imperviousness parameter and a value of 35 was set for the width parameter (the fact that these are the same number are simply coincidence and did not occur for most of the pre and post-rehabilitation models). The effective area (WAREA), which affects both the surface flow and subsurface flows (groundwater), became the major calibration factor (i.e. the effective area of the basin is reduced through removing I/I sources). 
Once the inconsistency was corrected, the difference in effective area (WAREA) was approximately $27 \%$ between the pre and post-rehabilitation models. This reduction translates to a reduction of $27 \%$ of the area contributing $\mathrm{I} / \mathrm{I}$ in the sewer basin (this percent reduction result is valuable on its own, but will not be expounded on in this chapter - see future papers from the authors). The results indicate that each model (pre and post) may not be "optimally" calibrated to each individual dataset, but by constraining certain parameters, the pre and post models are "best" calibrated for use in design event projections. It should be noted that Figures 18.4 and 18.5 are the calibration hydrographs after imperviousness and width were constrained.

By examining pre and post-rehabilitation model results to effectively calibrate both models, this analysis presents a unique approach in the scope of applying automatic calibration procedures. This does not assume that pre and post flows are necessary for a proper calibration (since this data is not usually available), but it does add to the body of knowledge regarding calibration of hydrologic models. The parameters estimated from the pre and post-rehabilitation simulations are summarized in Table 18.3. The resultant hydrographs for the March 1983 event are illustrated in Figure 18.6.

Table 18.3 Calibration results.

\begin{tabular}{cccc}
\hline Parameter & $\begin{array}{c}\text { Pre- } \\
\text { Rehabilitation } \\
\text { Hydrograph }\end{array}$ & $\begin{array}{c}\text { Post- } \\
\text { Rehabilitation } \\
\text { Hydrograph }\end{array}$ & $\begin{array}{c}\text { Percent } \\
\text { Difference }\end{array}$ \\
\hline Surface Water & & & \\
WAREA & 14.0 & 10.2 & $27 \%$ \\
IMPERV & 35 & 35 & $0 \%$ \\
WIDTH & 35 & 35 & $0 \%$ \\
Groundwater & 1.4 & 1.7 & $-21 \%$ \\
STG & 3.1 & 2.7 & $13 \%$ \\
BC & 2.9 & 1.5 & $48 \%$ \\
TW & 0.056 & 0.037 & $34 \%$ \\
A1 & 3.4 & 3.8 & $-12 \%$ \\
B1 & 0.001 & 0.001 & $0 \%$ \\
A2 & 0.9 & 3.8 & $-322 \%$ \\
B2 & & & \\
Objective Functions & & & -- \\
Sum of & & 28.66 & $2 \%$ \\
Residuals & 35.39 & 0.833 & $10 \%$ \\
ENs & 0.854 & 0.647 & $4 \%$ \\
EM & 0.717 & 0.843 & \\
R & 0.879 & & \\
\hline
\end{tabular}

${ }^{(1)}$ Sum of Residuals is not normalized to the length of the time series and longer time series will have a higher value. $E_{N s}, E_{M}$, and $R^{2}$ are normalized and do not depend on length of time series (a perfect fit would equal 1.0). 


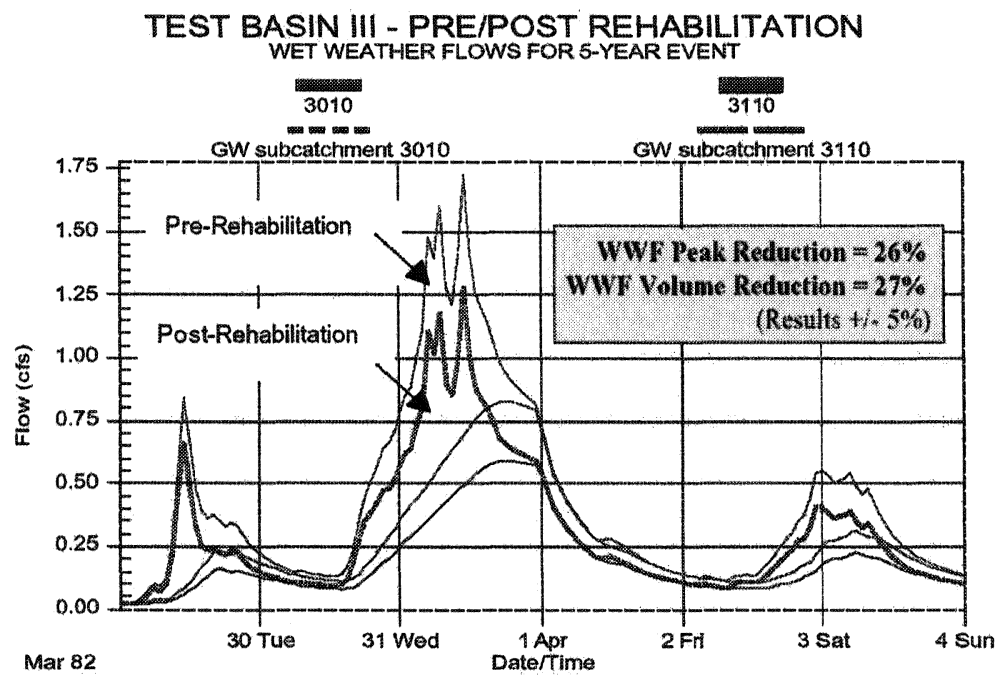

Figure 18.6 Pre- and post-rehabilitation hydrographs for a projected wet weather event (Carollo, 2003).

This exercise is very similar to the results that should be gained from a watershed study in an inverse manor. For example, flows in a watershed will increase over time if the watershed becomes more impervious (i.e. increasing surface runoff with more development). Accordingly, the post-development hydrograph should be greater than the pre-development hydrograph at each point in the time series. Based on the results of this analysis, it is recommended that this method be applied to a watershed, using pre and post development flows, to further examine the efficacy of this calibration method.

\subsubsection{Multiobjective Calibration}

Although this analysis did not apply multiobjective calibration procedures, it is worth some discussion on the benefits of using multiobjective principles in calibrating a model that projects $\mathrm{I} / \mathrm{I}$ in a sanitary sewer system. A single best parameter set that gives excellent results for one data set may perform very poorly for another (i.e. peak flows versus low flows). Due to the limitations in obtaining a globally optimal solution in calibrating a hydrologic model (as stated above), multi-objective calibration has garnered significant attention (Gupta et al., 1998; Madsen, 2000). 
Sum of Residuals is not normalized to the length of the time series and longer time series will have a higher value. ENS, EM, and R2 are normalized and do not depend on length of time series (a perfect fit would equal 1.0).

Multiobjective calibration can be simply stated as the simultaneous optimization of a number of different calibration objectives (Gupta et al., 1998, Madsen and Jacobsen, 2001). This process inherently includes a more rigorous calibration analysis than that accomplished through single objective calibration (which is primarily covered in this chapter). Calibration using multiobjective procedures benefits even more from automatic optimization techniques because of the increased complexity of the problem statement.

Model calibration, using a multiobjective approach, can be performed on the basis of multi-variable measurements (i.e. stage and flow), multi-site measurements (i.e. several flow measurements in a basin), and/or multiresponse modes (i.e. peak versus low flows), as described by Madsen and Jacobsen (2001). The solution, in a multiobjective analysis, will not be a single unique set of parameters, but will consist of a Pareto front of solutions. Choosing a single point along this front is commonly referred to as a pareto optimal or nondominant solution. For example, the chosen pareto optimal may not be the best solution when peak flows are considered, but a slight reduction in peak flow goodness-of-fit, as a trade off to improve the low flow estimates, are gained throughout a times series of flows.

\subsection{Conclusions and Recommendations}

This section identifies the conclusions from this analysis and also recommends further research that would add to the body of knowledge of not only automatic calibration but also modeling $\mathrm{I} / \mathrm{I}$ in sanitary sewer systems.

\subsubsection{Conclusions}

This chapter described the use of an automatic calibration routine using GA to estimate parameters for the SWMM Runoff model to calibrate I/I in a sanitary sewer system basin in Vallejo, California. Based on the results from this case study, the follow conclusions were made:

1. Error is introduced into the hydrologic modeling process in many ways. Uncertainty in measured data will ultimately affect the confidence in model calibration. Measurement error included rainfall (including rainfall variability) and flow measurement 
error. The inherent simplification of the physical hydrologic process also introduces error into the model, and thus the modeling processes. It is the modelers objective to minimize these errors whenever possible, but no hydrologic model i $\mathrm{s}$ ever error free.

2. Calibration of hydrologic models is necessary because of the errors noted above. The calibration process, of adjusting model parameters, has historically taken place through a trial and error approach. However examples of automated calibration for complex conceptual models are becoming more commonplace as advances in global metaheuristic search routines become more widespread. Estimations of goodness-of-fit should also be reported in any calibration procedure as an objective function. The objective functions reported in this study include RMSE (root mean square error), $\mathrm{E}_{\mathrm{NS}}$ (Nash-Sutcliffe coefficient of efficiency), $E_{M}$ (modified coefficient of efficiency), and $R^{2}$. The calibrations produced good results for these objective functions.

3. This study is one of the first of its kind to introduce an automatic calibration approach, using a GA and SWMM Runoff, to calibrate wet weather flow $(\mathrm{I} / \mathrm{I})$ in a sanitary sewer system. Evolver is an off-the-shelf GA optimization routine, which was coupled with a recompiled version of SWMM Runoff (as a DLL), to efficiently calibrate wet weather hydrographs for a single sanitary sewer basin under two different conditions (pre and post rehabilitation).

4. This study calibrated a pre and post rehabilitation model for a single sewer basin and then projected the results of these two models to a single design event. Although each model could be individually calibrated very well to each pre or post hydrograph, it was not until the models were used to project flow for a single design event that the efficacy of the calibration process could be tested. It was found that the parameter sets that were initially developed for the calibration of the separate models had to be adjusted since inconsistencies arose between the pre rehabilitation and post rehabilitation hydrographs (e.g. the receding limb of the pre rehabilitation hydrograph was initially projecting lower flows than the receding limb of the post rehabilitation hydrograph). These inconsistencies did not surface until the pre to the post hydrographs were analyzed using a single design event. This provided additional insight into the automatic calibration processes. 


\subsubsection{Recommendations}

The next major upgrade to SWMM (version 5) includes a DLL of all SWMM components, which will allow easy access to the model code as well as linkages to other programs (spreadsheets and/or optimization programs). It should also be noted that the Evolver program could be compiled into an executable standalone program that could be linked to SWMM (using the DLL structure) without having to use a spreadsheet as the interface. This would most likely further decrease the model run times as well as provide a standard interface for performing multiple model runs.

Further analysis should be completed to test other objective functions beyond the modified coefficient of efficiency used in this study. The use of multi-objective optimization should also be further explored within this model structure. The use of a Pareto front would provide an excellent means for comparing the results of model runs for peak flows versus total volume estimates. The automatic calibration method described herein would also be valuable when applied to the calibration of flows in pre and post developed watersheds and should be pursued in further research.

\section{Acknowledgments}

The authors would like to gratefully acknowledge Ron Matheson (General Manager) and RolfOhlemutz (Director of Engineering) of the Vallejo Sanitation and Flood Control District, and Professor James Heaney (University of Gainesville, Florida) for their support and guidance.

\section{References}

American Society of Civil Engineers and Water Pollution Control Federation (1983), Existing Sewer System Evaluation and Rehabilitation. ASCE Manuals and Reports on Engineering Practice 62, WPCF Manual of Practice FD-6.

Carollo Engineers (2003), Pilot Rehabilitation Program - Sanitary Sewer Overflow Elimination Program Technical Memorandum, Vallejo Sanitation and Flood Control District, CA.

Dent, S., Wright, L., Sathyanarayan, P., and Ohlemutz, R. (2003) "Identifying Where Rehabilitation is Cost-Effective in a Sanitary Sewer System to Manage SSOs." WEF Collection System Specialty Conference; cMOM Go!, Water Environment Federation, Austin, TX. 
Dent, S., Wright, L., Sathyanarayan, P., and Ohlemutz, R. (2002) "Developing A Wet Weather Design Event For A Collection System And WWTP." WEFTEC 2002, Collection System Symposium, Water Environment Federation, Chicago, IL.

Dent, S., Wright, L., Housen, V., and Mosley, C. (2000) Continuous simulation vs. design storm comparison with wet-weather flow prediction methods." Collection Systems Wet Weather Control: Looking into Public Private and Industrial Issues, Water Environment Federation, Rochester, NY.

Doherty, J., and Johnston, J.M. (2003) Methodologies for calibration and predictive analysis of a watershed model, J. of American Water Resources Association (JAWRA), 39(2):251-265.

Duan, Q., Sorooshian, S., and Gupta, H. (1992) Effective and efficient global optimization for conceptual rainfall-runoff models, Water Resources Resch, 28(4), 1015-1031.

Glover, F. and Laguna, M. (1997) Tabu search. Kluwer Academic Publishers, Boston, MA. 382 p.

Gupta, H.V., Sorooshian, S., and Yapo, P.O. (1999) Status of automatic calibration for hydrologic models: Comparison with multilevel expert calibration. ASCE Journal of Hydrologic Engineering, Vol. 4(2), 135-143.

Gupta, H.V., Sorooshian, S., and Yapo, P.O. (1998) Toward improved calibration of hydrologic models: Multiple and noncommensurable measures of information. Water Resources Research, 34(4), 751-763.

Hill, M.C. (1998) Methods and guidelines for effective model calibration, U.S. Geological Survey, Water Resources Investigations Report, 98-4005.

Huber, W.C., and Dickinson, R.E. (1988) Stormwater Management Model, Version 4: User's Manual. Cooperative Agreement CR-811607, U.S. Environmental Protection Agency, Athens, Georgia.

James, W., and James, R.C. (ed.) (2002) Water systems models [1] Hydrology. User's guide to SWMM4 Runoff and supporting modules (Rain, Temperature, Combine, and Statistics), Computational Hydraulics Institute, Guelph, ON.

James, W.R.C, Wan, B., and James, W. (2001) Implementation in PCSWMM using Genetic Algorithms for auto calibration and design-optimization. Proc. of the Urban Drainage Modeling Symposium, Portland, OR. ASCE, Reston, VA.

Khu, S.T. (1998) Automatic Calibration of NAM model with multi-objective consideration, D2K Technical Report 1298-1.

Legates, D.R. and McCabe, G.J. (1999) Evaluating the use of "goodness-of-fit" measures in hydrologic and hydroclimatic model validation. Water Resour. Res. 35(1):233-241.

Liong, S.Y., Chan, W.T., and Lum, L.H. (1991) Knowledge-based system for SWMM Runoff component calibration. ASCE Journal of Water Resources Planning and Management, 117(5),507-523.

Liong, S.Y., and Ibrahim, Y. (1994) Estimation of peak flow and runoff volume with response surface method. ASCE Journal of Water Resources Planning and Management, 120(2), 161-175. 
Liong, S.Y., Chan, W.T., and Shreeram, J. (1995) Peak-flow forcasting with genetic algorithm and SWMM. ASCE Journal of Hydraulic Engineering, 121(8), 613-617.

Madsen, H., and Jacobsen, T. (2001) Automatic calibration of the MIKE SHE integrated hydrological modeling system, 4th DHI Conference, Helsingor, Denmark.

Madsen, H. (2000) Automatic Calibration of the MIKE 11/NAM rainfall-runoff model, Nordic Hydrological Conference, Uppsala, Sweden, NHP-Report No. 46, Vol. 1, 276-283.

Nash, J.E. and Sutcliffe, J.V. (1970) River flow forcasting through conceptual models. I. A Discussion of Principles., J. Hydrol. 10:282-290.

Nguyen, V., H. Javaheri and S. Liong. 2001. "On Automatic Calibration of the SWMM Model." Journal of Water Management Modeling R206-09. doi: 10.14796/JWMM.R206-09.

Nix, S.J. (1994) Urban Stormwater Modeling and Simulation, CRC Press, Inc.

Palisade Corp. (2001). Evolver Excel Add-In. Palisade Corporation, Newfield, NY.

Sorooshian, S., Gupta, H.V., and Bastidas, L.A. (1998) Calibration of hydrologic models using multi-objectives and visualization techniques, Final Report on Project Proposal No. EAR-9418147, Dept. of Hydrology and Water Resources, The University of Arizona, Tucson, AZ.

Stonehouse, M.C., M.J. TenBroek, G. Fujita and T.J. Dekker. 2001. "An Installed Accuracy Assessment using Dye Dilution Testing for Seven Common Flow Metering Technologies." Journal of Water Management Modeling R207-18. doi: 10.14796/JWMM.R207-18.

Wan, B., James, W. (2001) Implementation in PCSWMM using Genetic A 1 g o rithms for auto calibration and design-optimization. Proc. of the Urban Drainage Modeling Symposium, Portland, OR. ASCE, Reston, VA.

Wangwongwiroj, N., Schlutter, F., and Mark, O. (2001) Application of an automatic calibration scheme for urban rainfall-runoff models in MOUSE, 4th DHI Conference, Helsingor, Denmark.

Wright, L.T. (2003) Design Optimization of a Sanitary Sewer Overflow Control Plan, $\mathrm{PhD}$ Thesis, Department of Civil, Architectural, and Environmental Engineering, University of Colorado, Boulder.

Wright, L.T., S. Dent, C. Mosley, P. Kadota and Y. Djebbar. 2001. "Comparing Rainfall Dependent Inflow and Infiltration Simulation Methods." Journal of Water Management Modeling R207-16. doi: 10.14796/JWMM.R207-16. 